\title{
KECK DEIMOS SPECTROSCOPY OF A GALEX UV-SELECTED SAMPLE FROM THE MEDIUM IMAGING SURVEY ${ }^{1}$
}

\author{
Ryan P. Mallery, ${ }^{2}$ R. Michael Rich, ${ }^{2}$ Samir Salim, ${ }^{2}$ Todd Small, ${ }^{3}$ Stephane Charlot,,${ }^{4}$ Mark Seibert ${ }^{3}$ \\ Ted Wyder, ${ }^{3}$ Tom A. Barlow, ${ }^{3}$ Karl Forster, ${ }^{3}$ Peter G. Friedman, ${ }^{3}$ D. Christopher Martin, ${ }^{3}$ \\ Patrick Morrissey, ${ }^{2}$ Susan G. NefF, ${ }^{6}$ David Schiminovich ${ }^{7}$, Luciana Bianchi, ${ }^{8}$ José Donas, $^{9}$ \\ Timothy M. Heckman, ${ }^{10}$ Young-Wook Lee, ${ }^{11}$ Barry F. Madore, ${ }^{12}$ Bruno Milliard, 9 \\ Alex S. Szalay, ${ }^{10}$ Barry Y. Welsh, ${ }^{13}$ and Sukyoung Y Y ${ }^{11}$ \\ Received 2006 January 25; accepted 2006 July 25
}

\begin{abstract}
We report results from a pilot program to obtain spectroscopy for objects detected in the Galaxy Evolution Explorer (GALEX) Medium Imaging Survey (MIS). Our study examines the properties of galaxies detected by GALEX fainter than the Sloan Digital Sky Survey (SDSS) spectroscopic survey. This is the first study to extend the techniques of Salim and coworkers to estimate stellar masses, star formation rates (SFRs), and the $b$ (star formation history) parameter for star-forming galaxies out to $z \sim 0.7$. We obtain redshifts for 50 GALEX MIS sources reaching NUV $=$ 23.9 (AB mag) having counterparts in the SDSS Data Release 4 (DR4). Of our sample, 43 are star-forming galaxies with $z<0.7,3$ have emission-line ratios indicative of active galactic nuclei with $z<0.7$, and 4 objects with $z>1$ are QSOs, 3 of which are not previously cataloged. We compare our sample to a much larger sample of $\sim 50,000$ matched GALEX/SDSS galaxies with SDSS spectroscopy; while our survey is shallow, the optical counterparts to our sources reach $\sim 3$ mag fainter in SDSS $r$ than the SDSS spectroscopic sample. We use emission-line diagnostics for the galaxies to determine that the sample contains mostly star-forming galaxies. The galaxies in the sample populate the blue sequence in the NUV $-r$ versus $M_{r}$ color-magnitude diagram. The derived stellar masses of the galaxies range from $10^{8}$ to $10^{11} M_{\odot}$, and derived SFRs are between $10^{-1}$ and $10^{2} M_{\odot} \mathrm{yr}^{-1}$. Our sample has SFRs, luminosities, and velocity dispersions that are similar to the samples of faint compact blue galaxies studied previously in the same redshift range by Koo and collaborators, Guzmán and collaborators, and Phillips and collaborators. However, our sample is $\sim 2$ mag fainter in surface brightness than the compact blue galaxies. We find that the star formation histories for a majority of the galaxies are consistent with a recent starburst within the last $100 \mathrm{Myr}$.
\end{abstract}

Subject headings: galaxies: active — galaxies: high-redshift — galaxies: starburst — ultraviolet: galaxies

\section{INTRODUCTION}

Following the successful launch and early operations of the $\mathrm{Gal}$ axy Evolution Explorer (GALEX) satellite in 2003, we decided to undertake an initial spectroscopic assay of sources detected by GALEX using the DEIMOS multiobject spectrograph (Faber et al. 2003) on the Keck II telescope. Although GALEX data have been matched to relatively deep optical surveys (Schiminovich et al. 2005), our study is the first to extend the modeling technique of Salim et al. (2005) from $z=0.25$ to $z \sim 0.5$.

The power of the GALEX Medium Imaging Survey (MIS) to select interesting objects is noteworthy. We recall that the MIS observations are $1500 \mathrm{~s}$ in duration, imaging FUV and NUV simultaneously (Martin et al. 2005a; Morrissey et al. 2005). The GALEX satellite has investigated the ultraviolet universe to

\footnotetext{
1 Some of the data presented herein were obtained at the W. M. Keck Observatory, which is operated as a scientific partnership among the California Institute of Technology, the University of California, and the National Aeronautics and Space Administration. The Observatory was made possible by the generous financial support of the W. M. Keck Foundation.

2 Department of Physics and Astronomy, University of California, Los Angeles, CA 90095-1562.

3 California Institute of Technology, MC 405-47, 1200 East California Boulevard, Pasadena, CA 91125.

4 Max-Planck Institut für Astrophysik, D-85748 Garching, Germany.

5 Institut d'Astrophysique de Paris, CNRS, 98 bis boulevard Arago, F-75014 Paris, France.

${ }^{6}$ Laboratory for Astronomy and Solar Physics, NASA Goddard Space Flight Center, Greenbelt, MD 20771.
}

$z \sim 1$ with the goal of determining, among other things, the star formation rate (SFR) of galaxies in the local universe, $z \lesssim 0.25$ (Salim et al. 2005, 2007; Martin et al. 2005b; Wyder et al. 2005; Treyer et al. 2005), and the evolution of the global star formation density out to $z \sim 1$ (Schiminovich et al. 2005). The ultimate goal of such work along with other surveys and observations of galaxies at similar and higher redshifts is to constrain the baryonic physics of galaxy formation and evolution. Questions still remain as to how the SFR depends on different factors such as environment, mass, and morphology (Martin et al. 2005a). Previous work deriving SFRs with a UV-selected sample was carried out by Sullivan et al. (2000), who derived SFRs both from UV luminosities at $2000 \AA$ and $\mathrm{H} \alpha$ luminosities from optical spectra for a sample of galaxies at redshifts of $0<z<0.4$ detected in the UV by the balloon-borne telescope FOCA (Milliard

\footnotetext{
${ }^{7}$ Department of Astronomy, Columbia University, New York, NY 10027.

${ }^{8}$ Center for Astrophysical Sciences, The Johns Hopkins University, 3400 North Charles Street, Baltimore, MD 21218.

${ }_{9}$ Laboratoire d'Astrophysique de Marseille, BP 8, Traverse du Siphon, 13376 Marseille Cedex 12, France.

${ }_{10}$ Department of Physics and Astronomy, The Johns Hopkins University, Homewood Campus, Baltimore, MD 21218.

${ }_{11}$ Center for Space Astrophysics, Yonsei University, Seoul 120-749, Korea.

12 Space Sciences Laboratory, University of California, Berkeley, 601 Campbell Hall, Berkeley, CA 94720; and Observatories of the Carnegie Institution of Washington, 813 Santa Barbara Street, Pasadena, CA 91101.

${ }_{13}$ Space Sciences Laboratory, University of California, Berkeley, 601 Campbell Hall, Berkeley, CA 94720.
} 

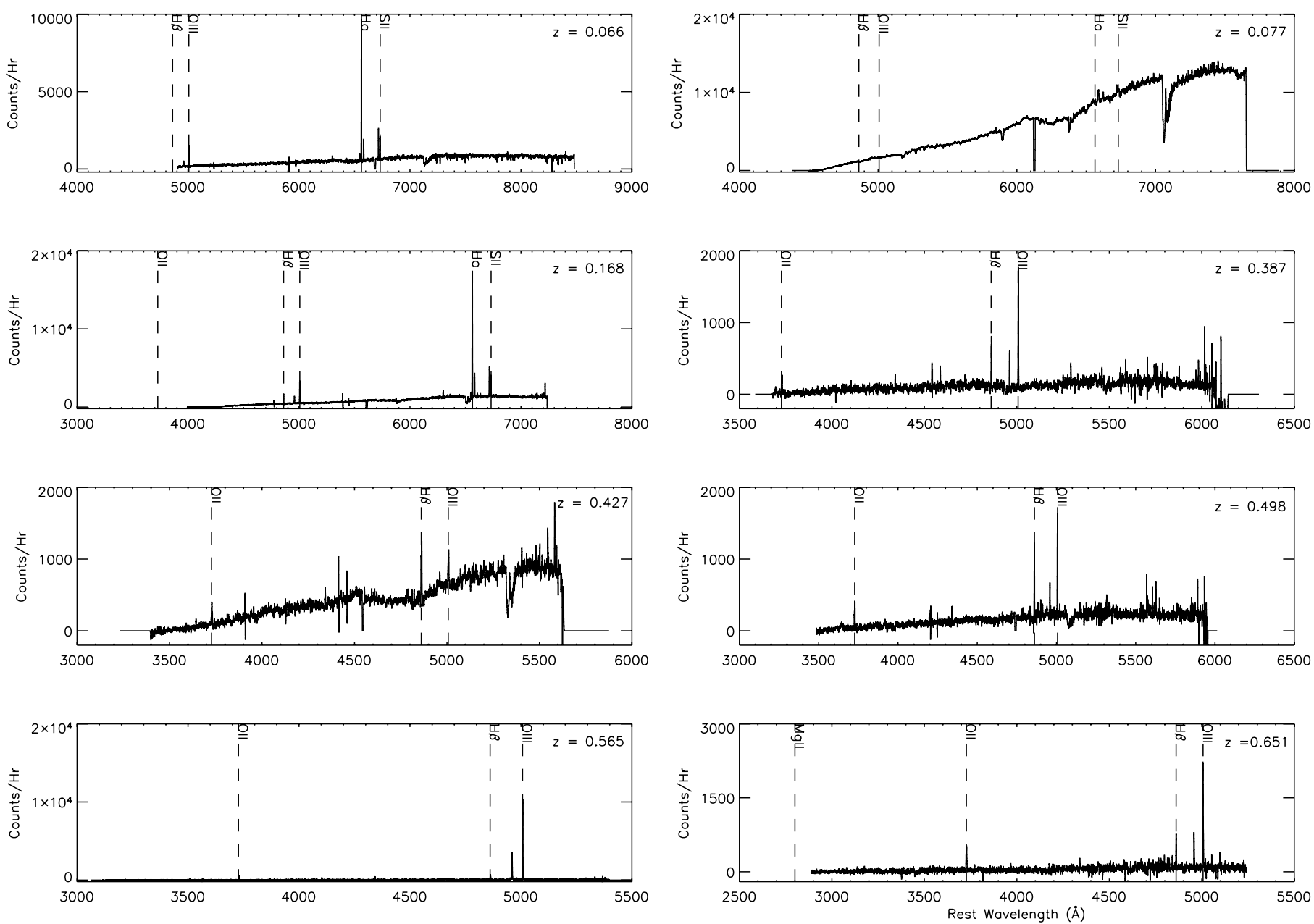

FIG. 1.- Spectra of several galaxies from our sample at a range of different redshifts. All of the galaxies are blue-sequence galaxies except the galaxy at $z=0.77$, which lies on the red sequence. The spectra have been boxcar smoothed by 5 pixels.

et al. 1992). Estimates of local SFRs before this were only possible through either optical emission-line luminosities of the recombination lines of hydrogen, mostly $\mathrm{H} \alpha$, and [O II] $\lambda 3727$ or the far-infrared luminosity from 10 to $100 \mu \mathrm{m}$ (Kennicutt 1998)

In this paper we present the observations and results from the pilot program for a sample of objects detected by GALEX and SDSS with spectra obtained from the DEIMOS spectrograph at the Keck II telescope. The analysis of this sample is enhanced greatly by the techniques of Salim et al. (2005) that have been expanded to include the derivation of galaxy physical parameters, such as stellar mass, from GALEX + SDSS photometry and redshifts. The main goal of this paper is to characterize the galaxies detected by GALEX that are fainter than SDSS Spectroscopic Galaxy Sample, in terms of mass, SFR, and UV - optical color. We are predominantly (about two-thirds of the sample) seeing the faint blue galaxy population (Ellis 1997) and are also sensitive to QSOs. While GALEX observations have been undertaken in many deep fields with much higher median redshifts (Schiminovich et al. 2005), we have the advantage in this sample of obtaining spectroscopy with Keck DEIMOS, which gives wavelength coverage sufficient to derive some detailed physical parameters from the spectra. In $\S 2$ we describe the sample and the photometric and spectroscopic data. Redshift and emission-line flux measurements from the spectroscopy are presented in $\S 3$. The distributions of UV color, UV-optical color, and the SDSS $r$ magnitude for the sample are described in $\S 4$. The derived galaxy parameters from spectral energy distribution (SED) fits are described in $\S 5$. We give a discussion and summary of our findings in $\S \S 6$ and 7. Throughout this paper we assume $H_{0}=70 \mathrm{~km} \mathrm{~s}^{-1} \mathrm{Mpc}^{-1}$, $\Omega_{m}=0.3$, and $\Omega_{\Lambda}=0.7$.

\section{SAMPLE AND OBSERVATIONS}

\subsection{GALEX/SDSS Data}

$G A L E X$ is a NASA Small Explorer Mission aimed to survey the UV emission from Galactic and extragalactic sources from $700 \mathrm{~km}$ circular orbit (Martin et al. 2005a; Morrissey et al. 2005). GALEX images the sky simultaneously in two bands, the far-UV (FUV,

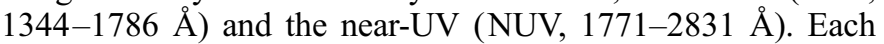
$G A L E X$ circular field is $1.25^{\circ}$ in diameter. We use FUV and NUV magnitudes and magnitude errors derived in elliptical apertures. ${ }^{14}$ The photometry is taken from the GALEX Internal Release 1.1 (IR1.1). The GALEXMIS photometry for tile number 10273 has a limiting magnitude, $m_{\text {lim }}(\mathrm{AB})=23$.

We use optical photometry for our objects obtained from SDSS Data Release 4 (DR4; Abazajian et al. 2004). The SDSS photometric data are taken with the $2.5 \mathrm{~m}$ telescope at Apache Point Observatory. Imaging is obtained in ugriz bands (Fukugita et al.

14 GALEX source detection and measurement is obtained from SExtractor (Bertin \& Arnouts 1996). 

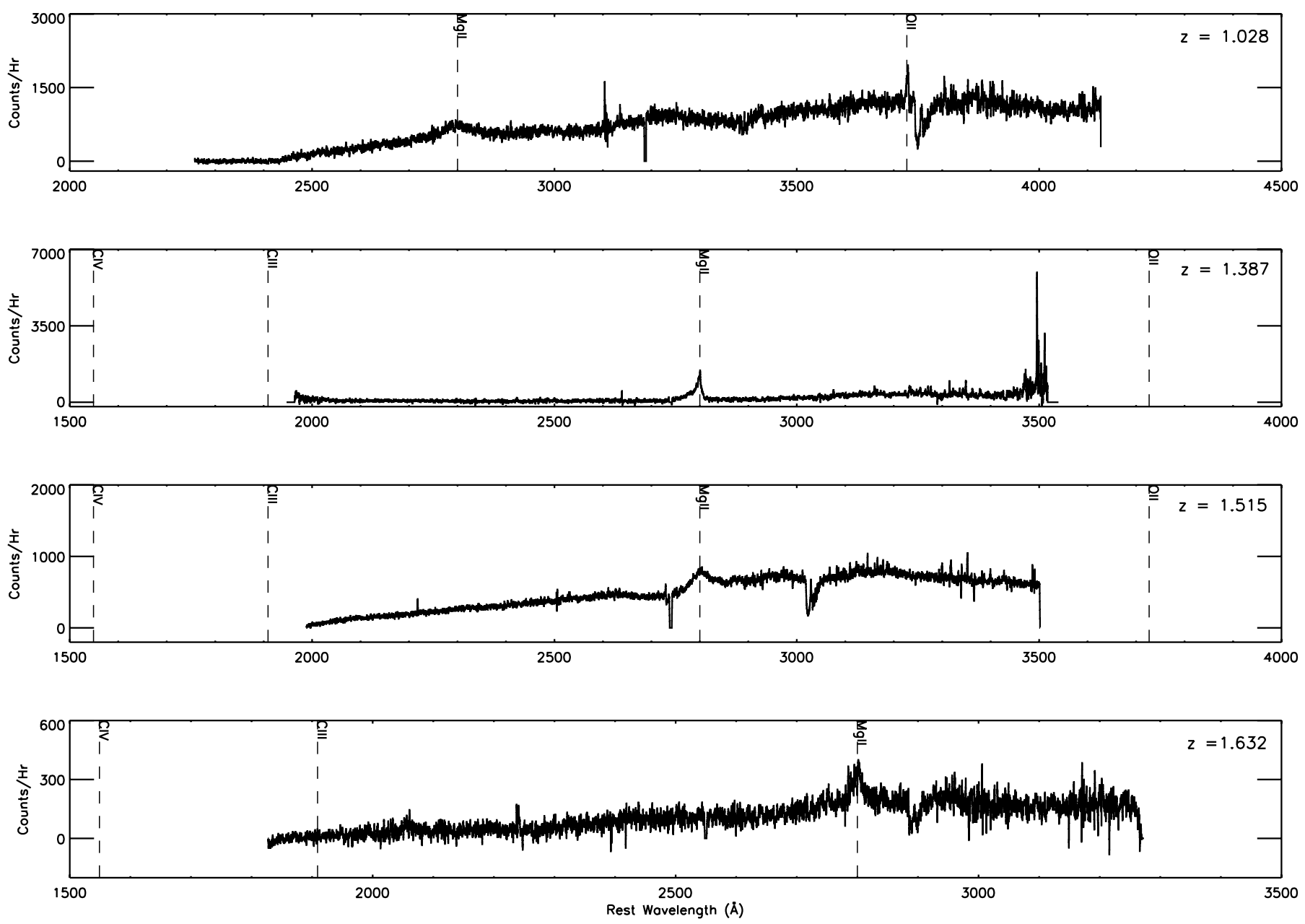

FIG. 2.- Spectra of four QSOs from our sample. The spectra have been smoothed by 5 pixels.

1996; Smith et al. 2002). The imaging data are photometrically (Hogg et al. 2001) and astrometrically (Pier et al. 2003) calibrated. An overview of the SDSS data pipelines and products can be found in Stoughton et al. (2002).

\subsection{Sample}

Our sample is derived from objects with detections in the GALEX MIS tile 10273, centered at a right ascension of $17^{\mathrm{h}} 40^{\mathrm{m}} 32.07^{\mathrm{s}}$ and a declination of $57^{\circ} 10^{\prime} 45.15^{\prime \prime}$. The GALEX field being much larger than the DEIMOS slit mask, we selected objects from the GALEX field to populate two DEIMOS slit masks. The two DEIMOS fields were initially chosen to maximize the number of MIS objects with optical counterparts in the SDSS classified as galaxies with FUV - NUV $<0$ photometry from an early version of the GALEX data reduction pipeline. The number of galaxies matching this criteria was much smaller than the number of available slits, and so to take full advantage of the DEIMOS field of view, the slits were populated by any galaxy detected by both GALEX and SDSS. In addition, the remainder of available slits in both fields was populated with blue stellar-like objects (QSOs and white dwarfs), main-sequence outlier stars $(u-g<1$ or $g-r<0$ ), and bright alignment stars. The first DEIMOS field contains 26 objects classified as galaxies in SDSS and 10 blue stellar objects and main-sequence outlier stars. The second DEIMOS field contains 19 objects classified as galaxies in SDSS and 9 blue stellar objects and main-sequence outlier stars.

\subsection{Spectroscopic Observations}

The spectroscopic data were obtained at the Keck II telescope with the DEIMOS multiobject spectrometer on 2003 October 1 (Faber et al. 2003). Spectra were obtained using two slit masks for a total 64 spectra. The 830 grooves $\mathrm{mm}^{-1}$ grating was used with a slit width of $0.73^{\prime \prime}$, giving a resolution of $\sim 2.5 \AA$ FWHM. The signal-to-noise ratio obtained over the continuum of each spectrum varied between sources from 1 to $\sim 20$, depending on the brightness of the source. The integration time for both slit masks was 30 minutes. Flux calibration of the spectra was not performed as no flux standards were observed. The spectra cover a wavelength range of $\sim 5000-9000 \AA$. Figure 1 shows a panel of several one-dimensional spectra for star-forming galaxies in the sample at a range of redshifts, plotted in the rest wavelength, and Figure 2 shows the spectra for objects with measured $z>1$.

\section{REDSHIFT DETERMINATION AND MEASUREMENT OF EMISSION LINES}

In all, we measured 50 redshifts, 45 for sources classified as galaxies in SDSS and five for sources classified as stars in SDSS. Of those five, three are QSOs at $z>1$ and the other two are starforming galaxies. The remaining 14 of the sources classified as stars by the SDSS have stellar spectra. The redshifts for objects with detected emission lines were measured by eye. For objects with $z<1$, multiple emission lines were detected including either

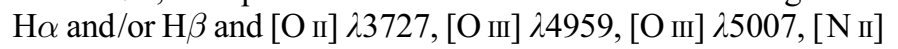




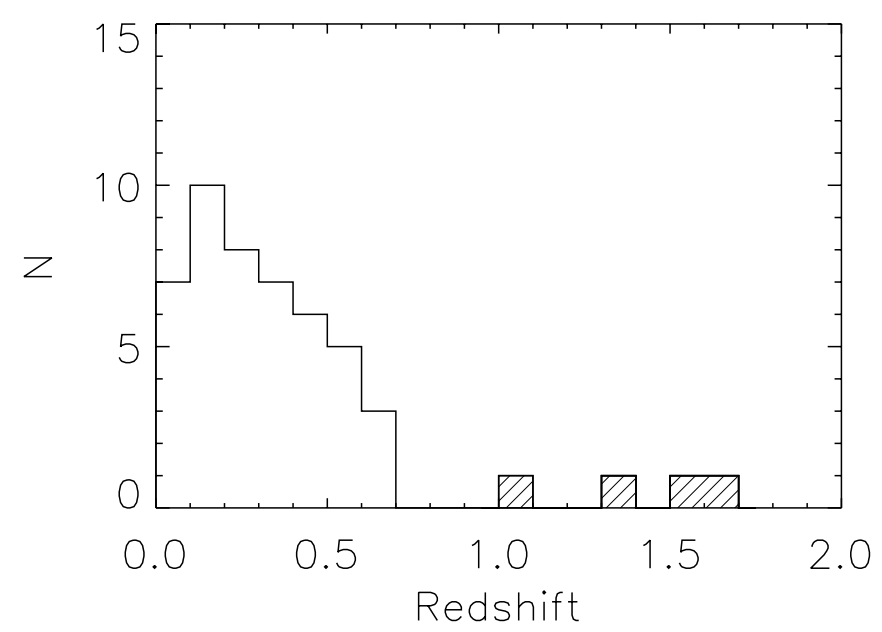

FIG. 3.-Redshift distribution of spectroscopic sample. The mean redshift for the sample is $z=0.421$. Shaded boxes indicate the quasar redshifts. $\lambda 6584$, [S II] $\lambda 6717$, and [S II] $\lambda 6731$. For all objects where the doublet [O II] $\lambda 3727$ was detected, $\mathrm{H} \alpha$ was redshifted beyond the wavelength range of the detector. For one source, at $z=1.028$, we detected two features that we assigned as [O II] $\lambda 3727$ and $\mathrm{Mg}$ II $\lambda 2800$ emission. For the other three sources with $z>1$ we find only one feature that we identify as Mg II $\lambda 2800$, based on the width of the line and the absence of other features in the spectrum. Figure 3 shows the redshift distribution of the sample. The mean redshift of the sample is $z_{\text {mean }}=0.421$. Two of the sources in the DEIMOS sample have corresponding SDSS spectra. The redshifts obtained for these two objects agree with the SDSS redshifts. One of the objects with SDSS spectra is the QSO at $z=1.028$, and the other is a galaxy at $z=0.176$. Both the DEIMOS spectra and the SDSS spectra for the latter object have significant detections of only $\mathrm{H} \alpha$ and [N II] $\lambda 6584$.

We measure spectral emission-line fluxes and equivalent widths for [O II] $\lambda 3727, \mathrm{H} \beta,[\mathrm{O}$ III] $\lambda 4959,[\mathrm{O}$ III] $\lambda 5007, \mathrm{H} \alpha$, [N II] $\lambda 6584$, [S II] $\lambda 6717$, and [S II] $\lambda 6731$. Fluxes, equivalent widths,

TABLE 1

Redshifts, Equivalent Widths, and Line Ratios for the Spectroscopic Sample

\begin{tabular}{|c|c|c|c|c|c|c|c|c|}
\hline SDSS Object ID & $z$ & $\mathrm{EW}_{[\mathrm{O} \text { пI]3727 }}$ & $\mathrm{EW}_{\mathrm{H} \beta}$ & $\mathrm{EW}_{[\mathrm{O} \text { 피 }] 5007}$ & $\mathrm{EW}_{\mathrm{H} \alpha}$ & $\mathrm{EW}_{[\mathrm{N} \mathrm{II]} 6584}$ & {$[\mathrm{O}$ III $] 5007 / \mathrm{H} \beta$} & {$[\mathrm{N} \mathrm{II}] 6584 / \mathrm{H} \alpha$} \\
\hline $587725578037494286 \ldots$ & 0.134 & 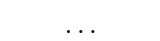 & $2.05 \pm 0.20$ & $2.57 \pm 0.22$ & $4.51 \pm 0.05$ & $3.46 \pm 0.13$ & $1.37 \pm 0.18$ & $0.25 \pm 0.01$ \\
\hline $587725578037495014 \ldots \ldots \ldots \ldots \ldots \ldots$ & 0.213 & $\ldots$ & $6.16 \pm 4.88$ & $4.83 \pm 4.78$ & $58.32 \pm 30.70$ & $3.91 \pm 16.30$ & $1.12 \pm 1.38$ & $0.98 \pm 0.60$ \\
\hline $587725578037494283 \ldots \ldots \ldots \ldots \ldots . . .$. & 0.084 & $\ldots$ & $0.82 \pm 0.25$ & $1.17 \pm 0.25$ & $2.36 \pm 0.05$ & $1.68 \pm 0.10$ & $1.84 \pm 0.69$ & $0.27 \pm 0.02$ \\
\hline 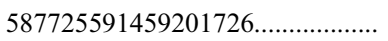 & 0.439 & $5.21 \pm 0.35$ & $3.17 \pm 0.18$ & $3.34 \pm 0.06$ & $\ldots$ & $\ldots$ & $4.95 \pm 0.30$ & \\
\hline $587725591459201436 \ldots$ & 0.066 & $\ldots$ & & $2.28 \pm 0.10$ & $2.25 \pm 0.03$ & $1.65 \pm 0.12$ & & $0.14 \pm 0.010$ \\
\hline $587725591459201670 \ldots$. & 0.651 & $6.22 \pm 0.58$ & $3.66 \pm 0.77$ & $5.06 \pm 0.21$ & & $\ldots$ & $3.98 \pm 0.84$ & \\
\hline $587725591459201656 \ldots \ldots$. & 0.157 & $\ldots$ & $2.04 \pm 0.51$ & $2.10 \pm 0.11$ & $2.53 \pm 0.12$ & $1.60 \pm 1.65$ & $5.81 \pm 1.47$ & $0.02 \pm 0.02$ \\
\hline $587725591459201666 \ldots$ & 0.078 & $\ldots$ & $1.03 \pm 1.80$ & $1.08 \pm 0.62$ & $2.10 \pm 0.11$ & $1.50 \pm($ & $2.36 \pm$ & $0.18 \pm 0.06$ \\
\hline $587725591459201614 \ldots$ & 0.188 & . & $2.62 \pm 0.40$ & $2.69 \pm 0.15$ & $3.38 \pm 0.10$ & $2.02 \pm($ & $3.01 \pm($ & $0.08 \pm 0.03$ \\
\hline $587725591459136404 \ldots$ & 0.220 & . & $0.74 \pm 0.59$ & $3.43 \pm 0$ & $4.95 \pm 0.23$ & $2.77 \pm 0.67$ & & $0.18 \pm 0.04$ \\
\hline 58772559145913635 & 0.319 & $1.34 \pm 1.16$ & $2.38 \pm 0$ & $0.76 \pm 0.45$ & & $\ldots$ & $0.34 \pm$ & \\
\hline 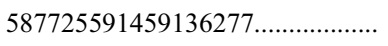 & 0.290 & $\ldots$ & & $1.81 \pm 1.07$ & $0.82 \pm 0.44$ & $2.80 \pm 0.41$ & & $3.97 \pm 2.21$ \\
\hline 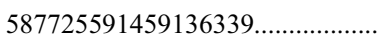 & 0.188 & $\ldots$ & $2.97 \pm 0.35$ & $2.69 \pm 0.35$ & $5.10 \pm 0.07$ & $5.15 \pm 0.17$ & $0.95 \pm 0.17$ & $0.36 \pm 0.01$ \\
\hline 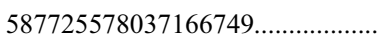 & 0.320 & $\ldots$ & $2.30 \pm 0.35$ & $2.45 \pm 0.86$ & $\ldots$ & $\ldots$ & $0.59 \pm 0.22$ & $\ldots$ \\
\hline 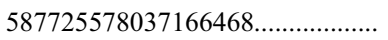 & 0.330 & $\ldots$ & $1.49 \pm 0.20$ & $0.94 \pm 0.17$ & $\ldots$ & $\ldots$ & $0.62 \pm 0.14$ & $\ldots$ \\
\hline 587725578 & 0.168 & $\ldots$ & $2.47 \pm 0.11$ & $3.06 \pm 0.07$ & $4.11 \pm 0.03$ & $3.06 \pm 0.09$ & $2.39 \pm 0.12$ & $0.20 \pm 0.01$ \\
\hline $587725578037166705 \ldots \ldots$. & 0.192 & $\ldots$ & $1.54 \pm 0.35$ & $2.71 \pm 0.29$ & $2.50 \pm 0.11$ & $1.24 \pm 0.50$ & $2.94 \pm 0.73$ & $0.06 \pm 0.03$ \\
\hline $587725578037166672 \ldots \ldots \ldots \ldots \ldots \ldots . .$. & 0.189 & $\ldots$ & $3.85 \pm 0.15$ & $2.92 \pm 0.20$ & $5.48 \pm 0.03$ & $5.96 \pm 0.07$ & $0.59 \pm 0.05$ & $0.34 \pm 0.01$ \\
\hline $587725578037166295 \ldots \ldots \ldots \ldots \ldots \ldots$ & 0.560 & $7.13 \pm 0.52$ & $5.94 \pm 0.38$ & $5.37 \pm 0.29$ & $\ldots$ & $\ldots$ & $1.29 \pm 0.11$ & $\ldots$ \\
\hline 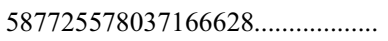 & 0.652 & $0.95 \pm 0.65$ & $3.54 \pm 0.43$ & $0.86 \pm 0.45$ & $\ldots$ & $\ldots$ & $0.17 \pm 0.09$ & $\ldots$ \\
\hline $587725578037166629 \ldots$ & 0.321 & $2.15 \pm 4.43$ & $2.93 \pm 0.17$ & $3.21 \pm 0.07$ & $\ldots$ & $\ldots$ & $3.02 \pm 0.19$ & $\ldots$ \\
\hline $587725578037100839 \ldots$ & 0.561 & $5.25 \pm 0.34$ & $2.84 \pm 0.15$ & $4.40 \pm 0.25$ & $\ldots$ & $\ldots$ & $1.70 \pm 0.13$ & $\ldots$ \\
\hline $587725578037101267 \ldots \ldots \ldots \ldots \ldots \ldots \ldots$ & 0.293 & & $7.99 \pm 8.21$ & $6.65 \pm 0.68$ & $2.60 \pm 1.68$ & $0.17 \pm 0.51$ & $\ldots$ & $0.10 \pm 0.30$ \\
\hline $587725578037101221 \ldots \ldots \ldots \ldots \ldots \ldots$ & 0.518 & $5.79 \pm 0.95$ & $3.31 \pm 0.35$ & $\ldots$ & $\ldots$ & $\ldots$ & $\ldots$ & $\ldots$ \\
\hline $587725578037166675 \ldots \ldots \ldots \ldots \ldots \ldots . .$. & 0.498 & $7.79 \pm 0.92$ & $3.15 \pm 0.23$ & $3.32 \pm 0.14$ & $\ldots$ & $\ldots$ & $1.39 \pm 0.11$ & $\cdots$ \\
\hline
\end{tabular}

${ }^{\text {a }}$ Denotes objects with corresponding spectra in SDSS.

b Denotes objects with failed SED fit. 


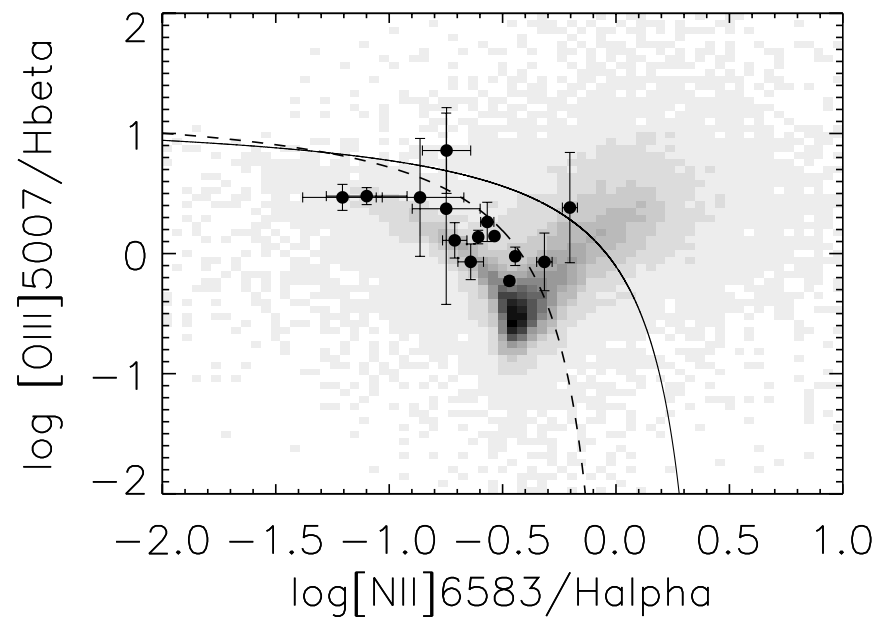

FIG. 4.-Emission-line diagnostic diagram first used by Baldwin et al. (1981). The shaded two-dimensional histogram corresponds a to the GALEX IR1.1/SDSS DR2 spectroscopic sample. The dashed curves taken from Kewley et al. (2001) and Kauffmann et al. (2003) show the distinction between sources with emission-line flux coming from AGN (above) and sources with emission line flux coming from $\mathrm{H}$ II regions (below). Out of the 14 objects from our DEIMOS sample with detections of $\mathrm{H} \beta$, [O III], $\mathrm{H} \alpha$, and [ $\mathrm{N}$ II] , only three have emission-line ratios consistent with emission due to an AGN.

and errors for the emission lines were measured in IDL using the MPFIT function. The continuum of each spectrum was first fit by a polynomial, and then the emission lines were simultaneously fit with Gaussians. Table 1 lists the objects, their equivalent widths, and flux ratios of [O III] $\lambda 5007 / \mathrm{H} \beta$ and [ $\mathrm{N} \mathrm{II}] \lambda 6584 / \mathrm{H} \alpha$ for objects with a $3 \sigma$ measurement of at least one of the above lines. We did not perform a reddening correction to the fluxes, since the standard procedure requires either detections of both $\mathrm{H} \alpha$ and $\mathrm{H} \beta$ or a measurement of the radio continuum (Osterbrock 1989). The effect of this on the flux ratios of [O III] $\lambda 5007 / \mathrm{H} \beta$ and [ $\mathrm{N} \mathrm{II}] \lambda 6584 / \mathrm{H} \alpha$ is thought to be negligible due to the small wavelength separation of the emission lines. In the DEIMOS sample we only have detections of both $\mathrm{H} \alpha$ and $\mathrm{H} \beta$ for 18 objects, all at $z<0.3$, and chose for consistency not to perform corrections on any of the spectra. We note that the foreground Galactic extinction, $E(B-V)$, calculated from the dust maps of Schlegel et al. (1998) is $\sim 0.05$ mag for the field.

Fourteen objects have measured fluxes of $\mathrm{H} \beta$, [O III $] \lambda 5007$, $\mathrm{H} \alpha$, and $[\mathrm{N}$ II] $\lambda 6584$. Figure 4 shows the emission-line diagnostic of Baldwin et al. (1981) used to distinguish star-forming galaxies and type II active galactic nuclei (AGNs). The solid curve is the boundary between star-forming galaxies and AGNs determined through modeling of starburst galaxy spectra by Kewley et al. (2001). A star-forming galaxy with only $\sim 20 \%$ of the optical emission-line flux due to an AGN would lie above the starforming/AGN boundary (Kewley et al. 2001). The dashed curve is the more stringent demarcation used by Kauffmann et al. (2003) to distinguish between AGNs and star-forming galaxies in SDSS. The shaded histogram shows the distribution of emission-line ratios for a sample of 51,000 SDSS/GALEX objects (see $\S 4$ ). The curve of Kauffmann et al. (2003) distinguishes between the two different sources of emission-line flux in this large sample, with the AGN occupying the parameter space to the right of the distribution of star-forming galaxies. In the DEIMOS sample only three galaxies of the 14 have emission-line flux ratios above this curve, implying that some portion of the flux is due to an AGN, although it is still within the errors that the emission-line flux for two of these objects results entirely from star-forming regions inside the host galaxies. Objects can also be AGNs if $\log \left[\mathrm{O}_{\mathrm{III}}\right] / \mathrm{H} \beta>1$ or

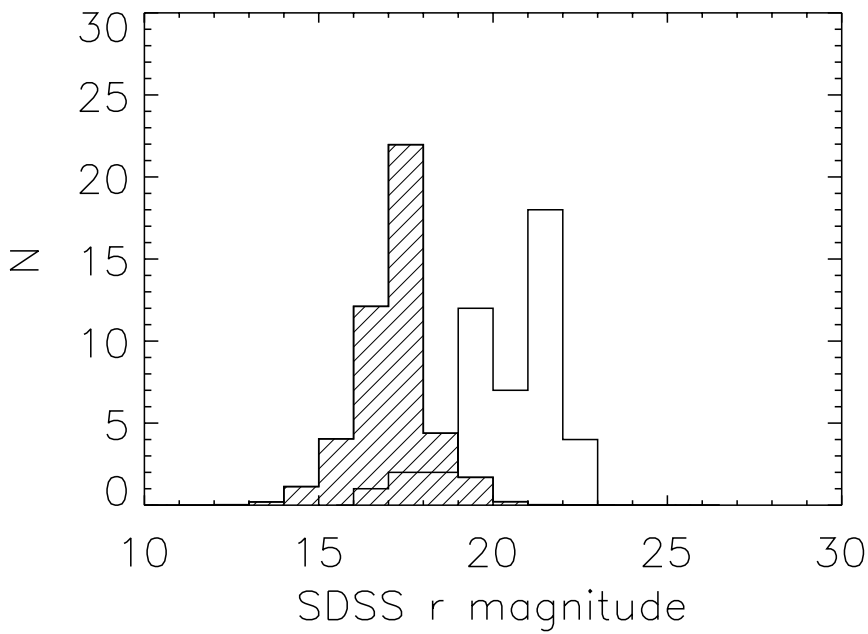

FIG. 5.- SDSS $r$-magnitude distribution of the DEIMOS spectroscopic sample plotted with the SDSS $r$-magnitude distribution of the matched GALEX IR1.1/SDSS DR2 spectroscopic sample (shaded histogram). The DEIMOS-matched spectroscopic sample mean $r$-magnitude (21) is 3 mag fainter than the mean of the SDSS spectroscopic sample.

$\log [\mathrm{N} \mathrm{II}] / \mathrm{H} \alpha>0.3$. Another 13 objects also have only detections of $\mathrm{H} \beta$ and [O III] $\lambda 5007$. Of the 13 , one has a flux ratio of $\log \left[\mathrm{O}_{\mathrm{III}}\right] / \mathrm{H} \beta>1$, indicative of an AGN. Sullivan et al. (2000) found a similar ratio of galaxies classified as star-forming/AGN from examination of emission lines in their sample of UV-selected galaxies.

\section{MAGNITUDE AND COLOR DISTRIBUTION}

The DEIMOS sample is $\sim 3$ mag deeper in $r$ than the SDSS spectroscopic sample. Figure 5 shows a histogram of the SDSS $r$ magnitudes for the sample plotted with the SDSS $r$ magnitude for GALEX MIS sources in IR1.1 with matches in the SDSS DR2 spectroscopic sample. The IR1.1/DR2 sample consists of $\sim 51,000$ galaxies at redshifts $0.005<z<0.25$ with derived star formation histories (SFHs) by SED fitting (see $\S 5$ ). The IR1.1/DR2 histogram is normalized to the size of the DEIMOS galaxy sample. The IR1.1/DR2 sample is mostly bounded in $r$ by the SDSS spectroscopic survey limits, $r<17.7$ (Strauss et al. 2002).

Using the redshifts determined for the DEIMOS sample we calculate the absolute $r$ magnitude, $M_{r}$, of galaxies in the sample, $k$-corrected to the mean redshift of the IR1.1/DR2 sample, $z=0.1$; $k$-corrections for all bandpasses were calculated using the publicly available code of Blanton et al. (2003), version 4.1.4. In Figure 6 we construct a color-magnitude diagram (CMD) of NUV $-r$ versus $M_{r}$. We overplot our sample onto the IR1.1/DR2 sample, plotted as a shaded contour plot. In the figure the diamonds correspond to DEIMOS objects with spectroscopically measured redshifts at $z<0.25$, and the crosses correspond to objects with spectroscopically measured redshifts at $z>0.25$. The contours enclose $40 \%$ and $80 \%$ of the objects in the IR $1.1 /$ DR 2 sample.

The CMD of the IR1.1/DR2 sample clearly shows the bimodality of galaxies seen by GALEX in the nearby universe, $z<0.25$, with distribution peaks at blue NUV $-r$ colors of $\sim 3$ and at red NUV $-r$ colors of $\sim 6$ (Wyder et al. 2005). We henceforth call these the blue and red sequences. The galaxies in the blue sequence are generally late-type in morphology and have spectra with emission line ratios indicative of star formation (Salim et al. 2005; Brinchmann et al. 2004), while the galaxies in the red sequence typically have absorption-line spectra and lower star formation rates than blue-sequence galaxies. The majority of the objects in the DEIMOS sample lie in the region of the diagram 


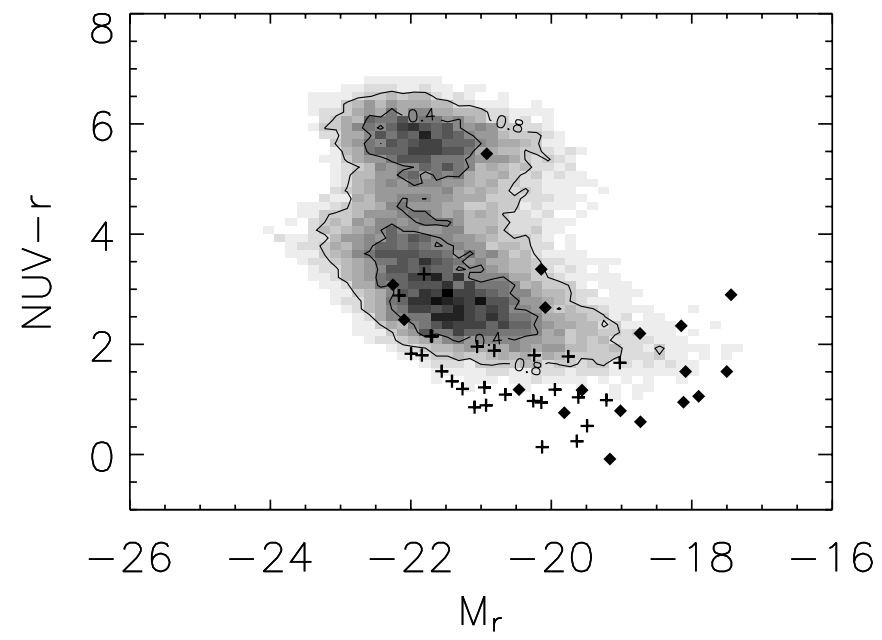

FIG. 6.- CMD of $M_{r}$ vs. NUV $-r$ plotted for the sample of GALEX/SDSS objects with DEIMOS spectroscopy plotted with the entire GALEX (IR1.1)/ SDSS (DR2) matched sample having SDSS spectroscopy. The diamonds represent galaxies in the DEIMOS sample at $z<0.25$; crosses represent galaxies in the DEIMOS sample at $z>0.25$. The IR1.1/DR2 sample is plotted as the shaded contour plot; the darker regions correspond to a higher density of points, and the contours encompass $40 \%$ and $80 \%$ of the of the objects in the sample. occupied by blue star-forming galaxies in the IR1.1/DR2 sample, mostly along the blue edge of the distribution, with only one having a NUV $-r$ color greater than 4 . The spectrum for this object, at $z=0.077$, is shown in Figure 1. The spectrum shows absorption features, $\mathrm{Na} \mathrm{D}$ and $\mathrm{Mg} \mathrm{I}$ b, but also shows weak emission features of $\mathrm{H} \alpha,[\mathrm{N}$ II] $\lambda 6584$, and [S II] $\lambda \lambda 6717,6731$.

In Figure 7 (bottom left) we again plot the CMD for the sample, highlighting the objects with FUV detections (filled circles). The other three panels of this figure show the UV color distribution for the 24 galaxies in the DEIMOS sample with FUV detections. Plotted for reference are galaxies from the IR1.1/DR2 sample with FUV detections shown as the shaded contour plots or shaded histograms in the respective panels. Besides the three galaxies in the DEIMOS sample with FUV - NUV $<0$ the UV color for this sample shows a similar distribution with the IR1.1/DR2 sample. In the FUV CMD the objects with $M_{r}<20$ show a range of UV color not seen in the local blue-sequence galaxies of similar optical luminosity. Both Figures 6 and 7 show that this sample probes the type of galaxies that one obtains when looking at objects detected by GALEX several magnitudes deeper than the SDSS spectroscopic limit.

\section{DERIVED GALAXY PARAMETERS}

We derive the following galaxy parameters according to the approach of Salim et al. (2005): the $V$-band dust attenuations, $A_{V}$ in magnitudes, stellar metallicity $Z$, the current SFR averaged over the past $100 \mathrm{Myr}$ in $M_{\odot} \mathrm{yr}^{-1}$, the present-day stellar mass, $M_{\star}$, of the galaxy in $M_{\odot}$, the fraction of stellar mass formed in bursts

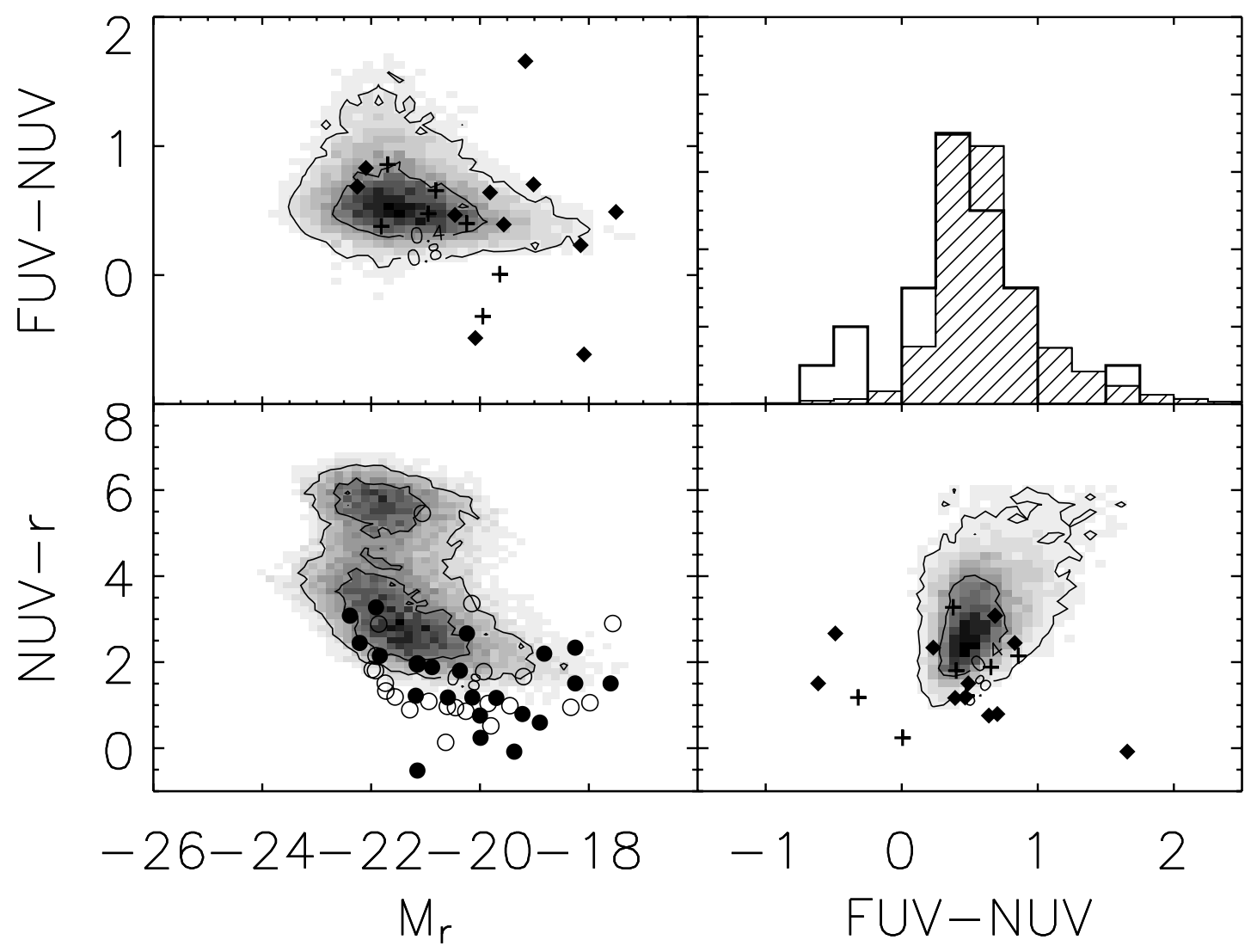

FIG. 7. - Top left: CMD of $M_{r}$ vs. FUV - NUV plotted for the 24 galaxies in the DEIMOS sample with FUV detections, plotted with objects in the IR1.1/DR2 matched sample with FUV detections, shown as a shaded contour plot. Top right: Histogram of FUV - NUV color for the 24 DEIMOS FUV galaxies plotted with distribution of the IR1.1/DR2 FUV sample (shaded histogram). Bottom left: Same as Fig. 6. The filled (unfilled) circles correspond to objects with (without) FUV detections. Bottom right: Color-color diagram of the FUV DEIMOS galaxies again overplotted onto the FUV IR1.1/DEIMOS sample. The symbols used are the same as in Fig. 6, and the contours encompass $40 \%$ and $80 \%$ of the of the objects in the IR $1.1 /$ DR 2 sample. 
TABLE 2

Galaxies, Redshifts, Colors, and Derived Parameters

\begin{tabular}{|c|c|c|c|c|c|c|c|c|c|}
\hline SDSS Object ID & $z$ & R.A. & Decl. & FUV & NUV & $\mathrm{NUV}-r$ & $\log M_{\star}$ & $\log \mathrm{SFR}$ & $\log b$ \\
\hline $587725578037166675 \ldots \ldots \ldots \ldots \ldots \ldots \ldots$ & 0.498 & 173734.409 & 572113.71 & $\ldots$ & 22.62 & 0.97 & 9.50 & 0.50 & -0.07 \\
\hline 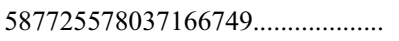 & 0.320 & 173741.177 & 571846.29 & 22.87 & 22.12 & 1.88 & 9.91 & 0.65 & -0.14 \\
\hline 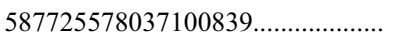 & 0.561 & 173743.542 & 57245.51 & & 22.19 & 1.19 & 10.40 & 0.85 & -0.32 \\
\hline 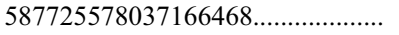 & 0.330 & 173745.923 & 571917.19 & 22.38 & 21.50 & 2.15 & 10.85 & 0.48 & -0.84 \\
\hline 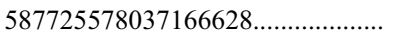 & 0.652 & 17382.622 & 572326.99 & $\ldots$ & 22.73 & 1.51 & 10.51 & 1.11 & -0.24 \\
\hline $587725578037166629 \ldots \ldots \ldots \ldots \ldots \ldots . .$. & 0.321 & 17387.756 & 572334.90 & $\ldots$ & 22.32 & 1.04 & 9.09 & 0.28 & -0.05 \\
\hline 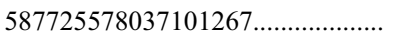 & 0.293 & 173822.192 & 57252.82 & $\ldots$ & 23.36 & 1.66 & 9.16 & -0.06 & -0.18 \\
\hline $587725578037100922 \ldots$ & 0.168 & 173823.628 & 572756.42 & 21.48 & 21.97 & 2.67 & 9.97 & -0.24 & -0.76 \\
\hline $587725578037101473 \ldots$ & 0.496 & 173827.876 & 572633.90 & 22.91 & 22.28 & 1.22 & 9.76 & 0.68 & -0.10 \\
\hline 587725578037101077..................... & 0.387 & 173836.526 & 573038.59 & $\ldots$ & 22.32 & 0.52 & 9.11 & 0.51 & -0.01 \\
\hline 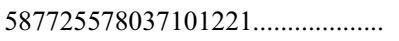 & 0.518 & 173837.317 & 572653.92 & $\ldots$ & 22.50 & 1.09 & 10.07 & 0.62 & -0.26 \\
\hline 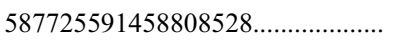 & 0.598 & 173854.873 & 57305.80 & $\ldots$ & 22.58 & 1.83 & 10.37 & 1.33 & -0.09 \\
\hline 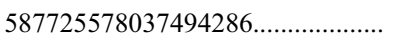 & 0.134 & 173945.205 & 56402.20 & 20.87 & 20.47 & 1.17 & 9.66 & -0.15 & -0.28 \\
\hline 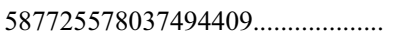 & 0.355 & 173951.035 & 563913.11 & $\ldots$ & 23.23 & 1.78 & 9.14 & 0.36 & -0.04 \\
\hline 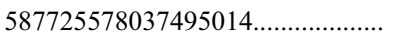 & 0.213 & 173951.343 & 563958.89 & $\ldots$ & 22.73 & 0.95 & 8.18 & -0.66 & -0.02 \\
\hline 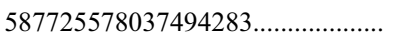 & 0.084 & 173953.130 & 564018.91 & 22.24 & 22.00 & 2.34 & 9.17 & -0.57 & -0.35 \\
\hline 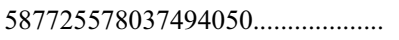 & 0.077 & 173956.243 & 563817.20 & $\ldots$ & 22.11 & 5.46 & 10.76 & -0.80 & -1.91 \\
\hline 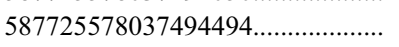 & 0.356 & 173956.287 & 563721.00 & $\ldots$ & 22.20 & 1.96 & 9.99 & 1.14 & -0.03 \\
\hline 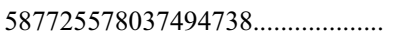 & 0.474 & 173956.704 & 563753.80 & $\ldots$ & 22.72 & 0.86 & 9.67 & 0.38 & -0.16 \\
\hline 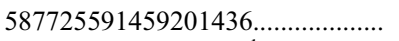 & 0.066 & 17401.663 & 56443.12 & 20.89 & 20.74 & 2.20 & 9.21 & -0.41 & -0.35 \\
\hline $587725591459201125^{\mathrm{b}} \ldots \ldots \ldots \ldots \ldots \ldots$ & 0.066 & 17403.853 & 564159.82 & 20.37 & 19.99 & 1.96 & $\ldots$ & $\ldots$ & $\ldots$ \\
\hline $587725591459201656 \ldots \ldots \ldots \ldots \ldots \ldots . .$. & 0.157 & 17406.724 & 564349.51 & 22.64 & 22.12 & -0.52 & 8.68 & -0.97 & -0.24 \\
\hline $587725591459201627 \ldots \ldots \ldots \ldots \ldots \ldots . .$. & 0.079 & 17408.196 & 564457.80 & 22.18 & 21.68 & 1.51 & 8.48 & -0.61 & -0.09 \\
\hline 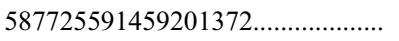 & 0.615 & 17408.328 & 563939.82 & $\ldots$ & 22.68 & 1.80 & 10.53 & 0.81 & -0.51 \\
\hline $587725591459136024^{\mathrm{b}} \ldots \ldots \ldots \ldots \ldots \ldots \ldots$ & 0.245 & 174032.498 & 564912.51 & 22.73 & 20.99 & -0.08 & $\ldots$ & $\ldots$ & $\ldots$ \\
\hline 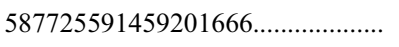 & 0.078 & 174033.193 & 56445.32 & $\ldots$ & 20.82 & 1.06 & 9.00 & -0.90 & -0.42 \\
\hline 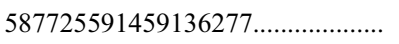 & 0.290 & 174035.691 & 565029.29 & 22.66 & 22.23 & 3.27 & 10.99 & 0.49 & -0.98 \\
\hline 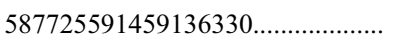 & 0.565 & 174046.362 & 564913.40 & $\ldots$ & 22.18 & 1.33 & 10.03 & 1.06 & -0.08 \\
\hline 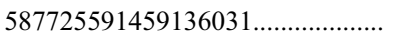 & 0.192 & 174056.177 & 564915.38 & 20.94 & 20.09 & 2.45 & 10.92 & 0.97 & -0.47 \\
\hline 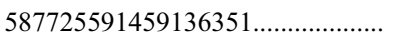 & 0.319 & 174056.594 & 564852.20 & $\ldots$ & 21.35 & 2.15 & 10.90 & 0.75 & -0.60 \\
\hline 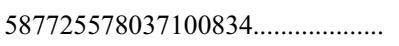 & 1.515 & 17389.360 & 572521.36 & $\ldots$ & 23.40 & 2.63 & $\ldots$ & $\ldots$ & $\ldots$ \\
\hline $587725591459201473 \ldots \ldots \ldots \ldots \ldots \ldots . .$. & 1.387 & 17409.836 & 56407.68 & $\ldots$ & 22.67 & 1.14 & $\ldots$ & $\ldots$ & $\ldots$ \\
\hline 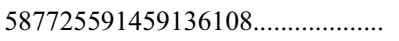 & 1.632 & 174043.682 & 564845.36 & $\ldots$ & 23.05 & 1.67 & $\ldots$ & $\ldots$ & $\ldots$ \\
\hline 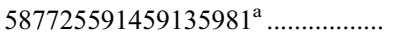 & 1.028 & 174049.197 & 564723.65 & 22.62 & 21.20 & 1.94 & $\ldots$ & $\ldots$ & $\ldots$ \\
\hline
\end{tabular}

Noте.-Units of right ascension are hours, minutes, and seconds, and units of declination are degrees, arcminutes, and arcseconds.

a Denotes objects with corresponding spectra in SDSS.

${ }^{b}$ Denotes objects with failed SED fit.

over the last $100 \mathrm{Myr}, F_{\text {burst }}$, and the Scalo (1986) $b$-parameter, defined as the ratio of the current SFR to the past time-averaged SFR (averaged over the estimated age, not Hubble time). The galaxy parameters are derived from model libraries of galaxies at redshifts between 0.1 and 1.6 at redshift increments of 0.1 for the DEIMOS sample, and at redshifts of $0.05,0.1,0.15,0.2$, and 0.25 for the IR1.1/DR2 sample. Each library consists of up to $\sim 10^{5}$ models. Each model is parameterized according to galaxy age, optical depth, $\mathrm{SFH}$, and metallicity. The SFH of each model is parameterized according to Kauffmann et al. (2003) with an underlying, continuous, exponentially declining SFR on which bursts of star formation, random in time and amplitude, are superimposed. Dust attenuation in each model is parameterized using the prescription of Charlot \& Fall (2000) using an effective $V$-band optical depth $\tau_{V}$ and absorption curve, $\tau \propto \lambda^{0.7}$, resulting from both giant molecular clouds and the diffuse interstellar medium (ISM), with the fraction $\mu$ of $\tau_{V}$ contributed only by the diffuse ISM. The $\mathrm{V}$-band optical depth from giant molecular clouds is taken to only 


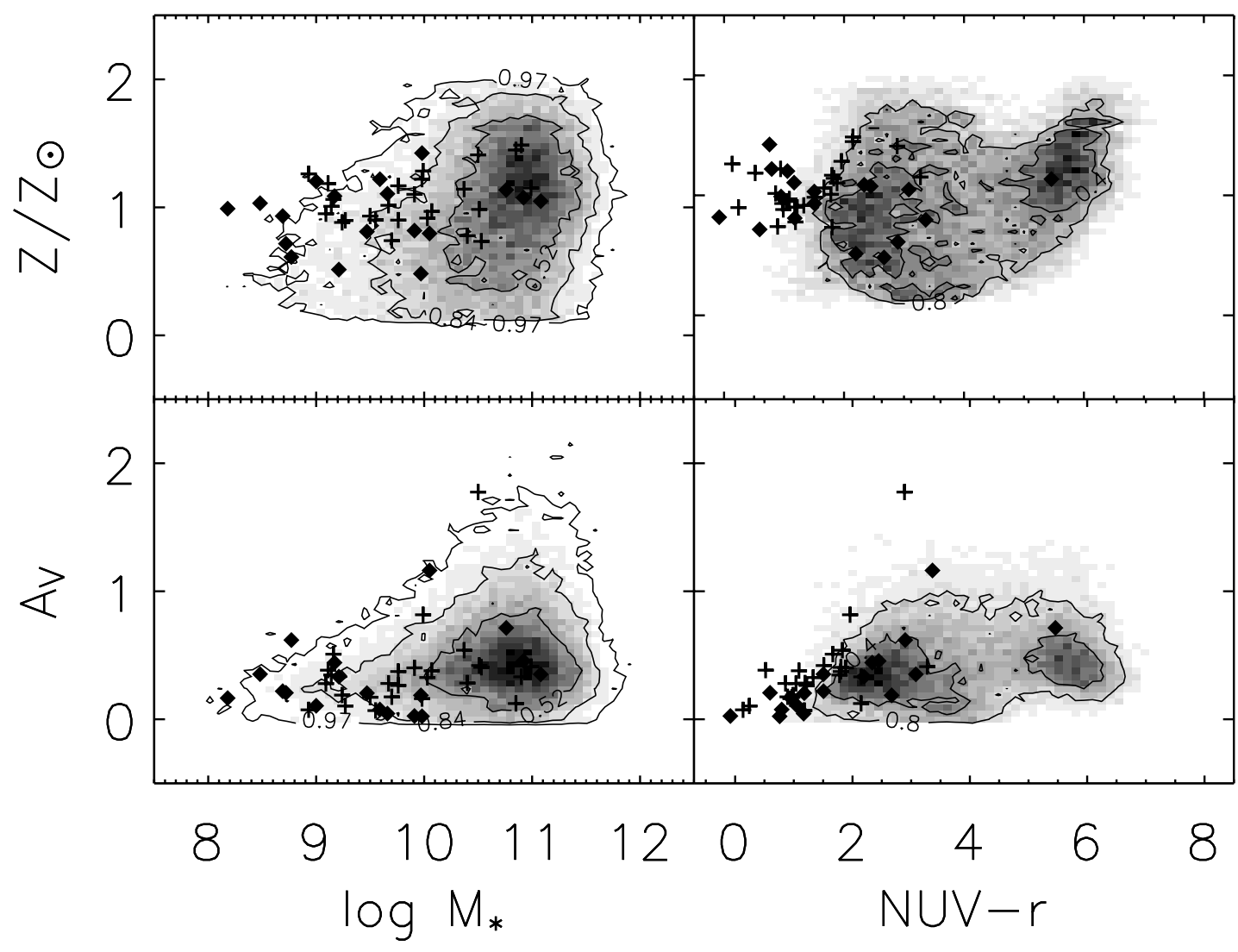

FIG. 8. -Derived galaxy parameters, metallicity, and $V$-band attenuation plotted vs. $M_{\star}$ and NUV $-r$ color. The DEIMOS spectroscopic sample is plotted over the matched GALEX IR1.1/SDSS DR2 spectroscopic sample (shaded contour plot). The crosses correspond to objects with redshifts $z>0.25$. The diamonds correspond to objects with redshifts $z<0.25$. The contours for the plots of stellar mass contain $57 \%, 84 \%$, and $97 \%$ of the data. The contours of NUV $-r$ color enclose $40 \%$ and $80 \%$ of the sample.

affect stars younger than 10 Myr. A description of the prior distributions of the model parameters is discussed in Salim et al. (2005).

SEDs are created for each galaxy in the library using the population-synthesis code of Bruzual \& Charlot (2003). The model SEDs are convolved with the GALEX and SDSS filterresponse curves. Statistical estimates of physical galaxy parameters are derived by comparing the observed seven-band GALEX/ SDSS fluxes of each galaxy to all the convolved model SEDs in the nearest redshift library. Probability density functions (PDFs) for each physical parameter are created by assigning weights to the parameters. The $\chi^{2}$ goodness offit of the model determines the weight $\left(\propto \exp \left[-\chi^{2} / 2\right]\right)$ that is assigned to the parameters of that model. The median (or most typical parameter value) of the PDF is taken as the estimate of the galaxy parameter.

We perform SED fits for galaxies not identified as QSOs. In Table 2 we list the galaxies, their redshifts, NUV $-r$ non$k$-corrected colors, and their derived parameters. The parameters derived from the SED fits are not used unless the reduced $\chi^{2}$ fit of at least one model is below 10. Only two of the 46 galaxies do not meet this criterion. Figures 8 and 9 show the derived parameters for the DEIMOS sample of galaxies overplotted on the IR1.1/DR2 sample plotted as shaded contour plots. The contours are labeled and encompass $52 \%, 84 \%$, and $97 \%$ of the data. The diamonds as before represent objects with $z<0.25$, and the crosses represent objects with $z>0.25$. The objects span the range of derived parameters of the IR1.1/DR2 sample for $Z$ and $A_{V}$.

The derived metallicities, $Z=[\mathrm{Fe} / \mathrm{H}]$, for the sample are not very well constrained. The typical error, $1 / 4$ of the difference between the 97.5 percentile and the 2.5 percentile of the PDF, of the derived metallicities is $\pm 43 \% Z_{\odot}$. The derived $M_{\star}$ range from $10^{8}$ to $10^{11} M_{\odot}$ with an average error of $\sim \pm 0.2 \mathrm{dex}$, but the majority of the $M_{\star}$ are at or below $10^{10} M_{\odot}$, about an order of magnitude below the mean of the IR1.1/DR2 sample. The derived SFRs lie between $10^{-1}$ and $10^{2} M_{\odot} \mathrm{yr}^{-1}$, with five galaxies having derived SFRs greater than $10 M_{\odot} \mathrm{yr}^{-1}$. The average error on the derived SFRs is typically \pm 0.3 dex. Comparing the SFRs between galaxies with similar NUV $-r$ color or $M_{\star}$, the galaxies at $z>0.25$ have SFRs about an order of magnitude greater than the galaxies at $z<0.25$. This is mostly a selection effect since the more distant galaxies are also more luminous on average. A comparison of these SED-derived SFRs with $\mathrm{H} \alpha$-derived SFRs cannot be performed since the spectra are not flux calibrated, and many lack $\mathrm{H} \alpha$. The range of SFRs we find for this sample is similar to the range of SFRs for local UV galaxies found by Sullivan et al. (2000) derived both from $\mathrm{H} \alpha$ luminosity and from the UV luminosity, but is an order of magnitude larger than the SFRs found by Kobulnicky \& Kewley (2004) in their sample of GOODS-North Treasury Keck Redshift Survey galaxies at $0.4<$ $z<.9$ when compared to galaxies in the DEIMOS sample with similar redshifts.

We find that the galaxies are largely starbursts with $\log b \sim$ -0.1 . This is shown in Figure 10. The distribution of $\log b$ for objects in the DEIMOS sample with SED fits are plotted with the distribution for the IR1.1/DR2 sample normalized to the number of objects in the DEIMOS sample with SED fits. The DEIMOS sample shows a highly peaked value of $\log b$ compared to the IR1.1/DR2 sample which has two small peaks near $\log b \sim-3$ and $\sim-1$, showing that most of these galaxies currently have less star formation now than in the past. The value $\log b \sim-0.1$ 


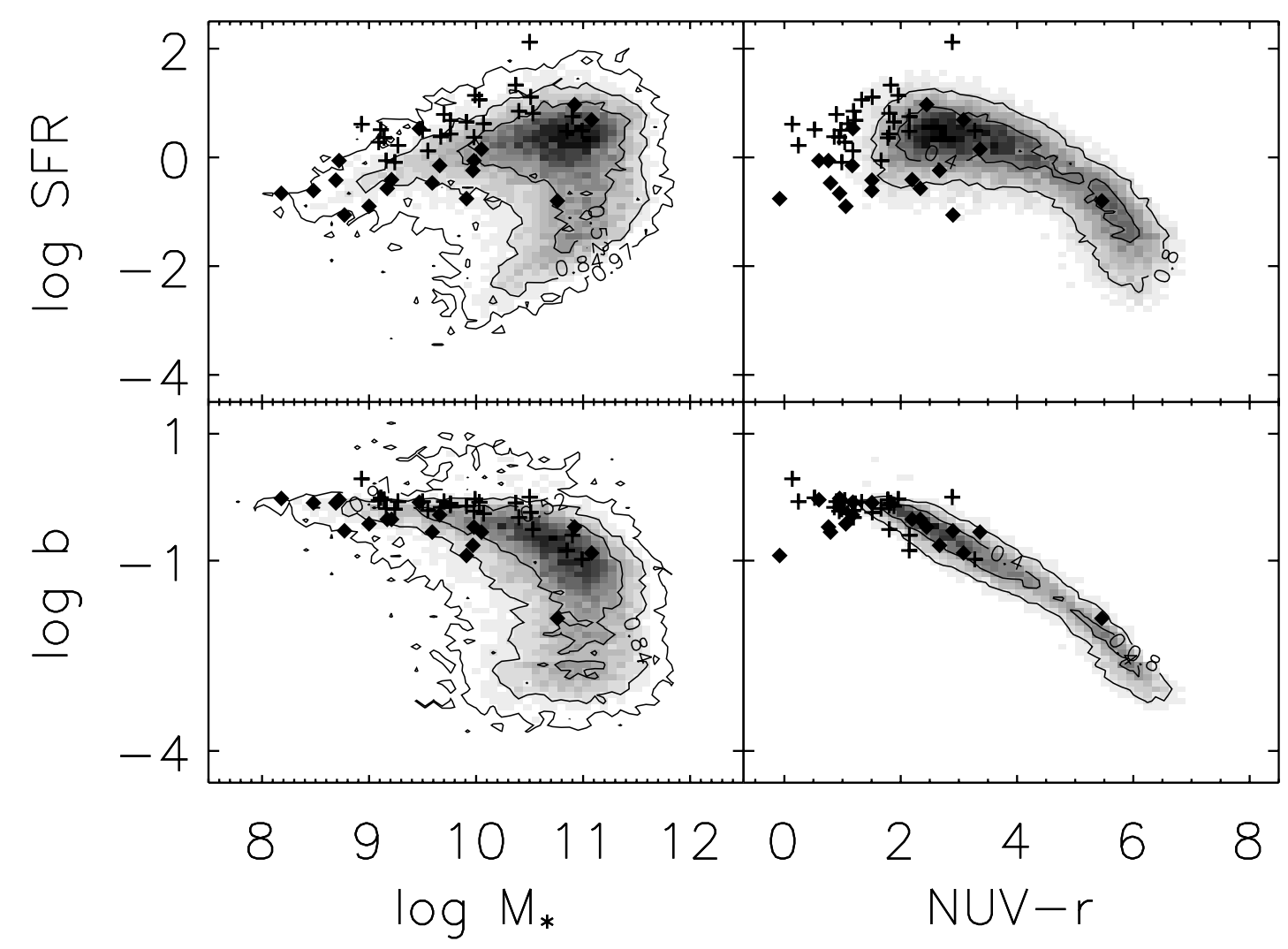

FIG. 9.-Derived Galaxy parameters, $\log$ SFR and $\log b$ plotted vs. $M_{\star}$ and NUV $-r$ color. The DEIMOS spectroscopic sample is plotted over the matched GALEX IR1.1/SDSS DR2 spectroscopic sample (shaded contour plot). The crosses correspond to objects with redshifts $z>0.25$. The diamonds correspond to objects with redshifts $z<0.25$. The contours for the plots of $M_{\star}$ contain $56 \%, 84 \%$, and $97 \%$ of the data. The contours of NUV $-r$ color enclose $40 \%$ and $80 \%$ of the data. The SFRs for the high- $z$ galaxies are approximately and order of magnitude higher than the SFRs of the low-z galaxies.

that describes most of the galaxies in the DEIMOS sample indicates that while these galaxies have less star formation than in the past, they are currently or have recently gone through a burst of star formation. Two of the galaxies have $\log b>0$, indicating that these galaxies are going through major starbursts in their histories. From a statistical standpoint, the SED fits reveal with a $95 \%$ reliability that at least three galaxies have not had a burst of

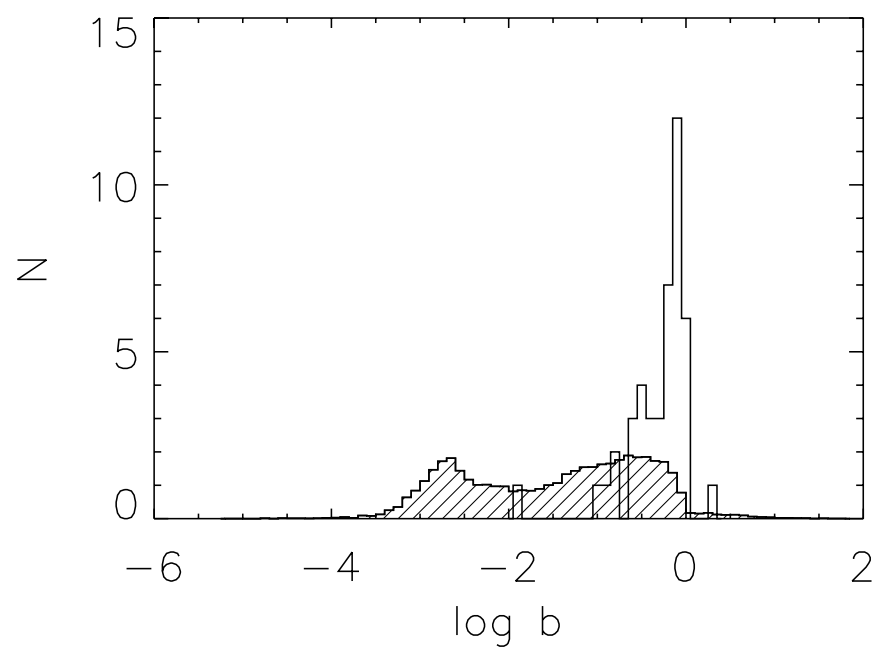

FIG. 10.-The $\log b$ histogram distribution of the DEIMOS sample plotted with the $\log b$ distribution of the matched GALEX IR1.1/SDSS DR2 spectroscopic sample (shaded histogram) normalized to the size of the DEIMOS sample. This figure reflects our predominant sensitivity to the blue-sequence star-forming galaxies. star formation in the last $100 \mathrm{Myr}$. Half of the galaxies in the sample could have formed as much as $\sim 10 \%$ of their stellar mass in a burst within the last $100 \mathrm{Myr}, 10$ could have formed up to $\sim 25 \%$, and five of the less massive systems could have had up to $\sim 50 \%$ of their stellar mass form in bursts within the last $100 \mathrm{Myr}$.

\section{DISCUSSION}

In the plot of NUV $-r$ versus $M_{r}$ our sample lies along a "blue sequence" of star-forming galaxies, and they have among the bluest colors for star-forming galaxies in the local universe found by GALEX and SDSS. While all the galaxies show blue NUV $-r$ color, this is not a homogenous sample. Fifteen of the galaxies show disk structure in the SDSS images. The remaining twothirds of the sample fit into the heterogeneous class of faint blue galaxies at intermediate redshifts previously studied by Koo et al. (1995), Guzmán et al. (1996, 1997, 1998) and Phillips et al. (1997) in the Hubble Deep Field and adjoining fields, and by Hammer et al. (2001) and Mallen-Ornelas et al. (1999) in the CanadaFrance Redshift Survey.

The faint blue subset of the DEIMOS sample has similar luminosities, SFRs, and optical colors to the compact galaxies found in the Hubble Deep Field by Phillips et al. (1997), although none are "compact" (optical half-light radii $<0.5^{\prime \prime}$ ). This is a selection effect in our sample. Galaxies with half-light radii this small are classified as stars in SDSS and would not have been selected for spectroscopy in our sample. In Figure 11 we plot, as diamonds, the rest-frame absolute $B$ magnitude versus the $B$ magnitude surface brightness and SFR versus velocity dispersion. The absolute $B$ magnitudes are calculated from the observed bandpasses using the $k$-correction code of Blanton et al. (2003), and the surface 

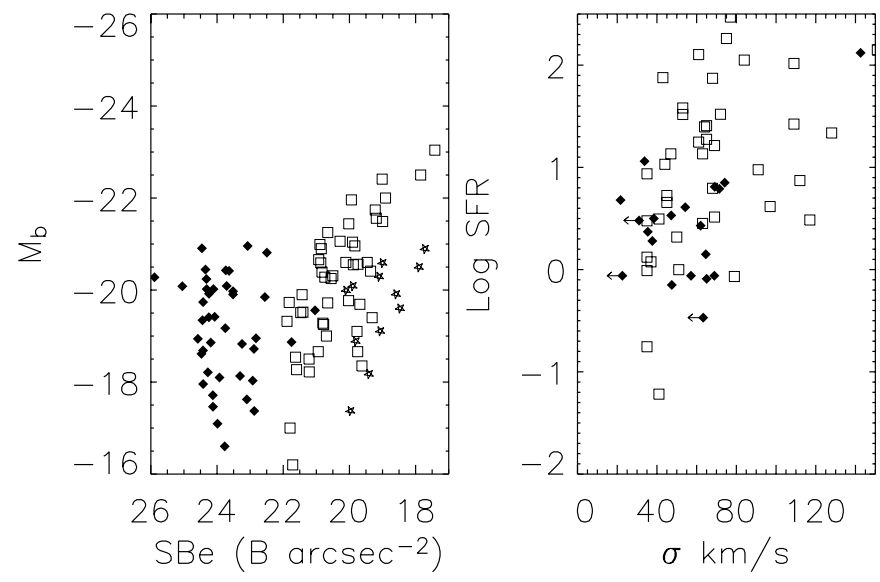

FIG. 11.-Left: Rest-frame absolute $B$ magnitude, $M_{b}$, plotted vs. the $B$ magnitude surface brightness within the Petrosian half-light radius $r$. Right: SFR vs. measured velocity dispersions. The diamonds correspond to objects in our sample. Objects taken from Koo et al. (1995) and Guzmán et al. (1996) are plotted as stars, and objects taken from Phillips et al. (1997) are plotted as squares. While the present sample has SFRs, luminosities, and velocity dispersions similar to the previous studies of faint blue galaxies, our sample contains sources with more extended emission and thus has lower surface brightness.

brightnesses are calculated with the half-light SDSS $r$ Petrosian radii. The velocity dispersions are derived from the measured line widths of [O III] $\lambda 5007$ for objects with $\geq 3 \sigma$ detections of the emission line. For comparison, we also plot, as stars and squares, the faint blue galaxy samples of Koo et al. (1995), Guzmán et al. (1996), and Phillips et al. (1997), making the necessary corrections to our adopted cosmology. While our sample of galaxies have luminosities, SFRs, and velocity dispersions comparable with the previous samples, the size selection effect separates our sample from the previous samples in surface brightness.

Koo et al. (1995) and Guzmán et al. (1998) proposed that the subset of the faint blue population of galaxies with compact geometry (half-light radii $<0.5^{\prime \prime}$ ) and narrow emission lines $\left(\sigma<65 \mathrm{~km} \mathrm{~s}^{-1}\right)$ will fade to become dwarf spheroidals by $z=0$, while Hammer et al. (2001) claimed that the most luminous of these are too massive to become dwarf spheroidals and instead will become the bulges of spiral galaxies. The most massive intermediate redshift galaxies $\left(M_{\star} \gtrsim 10^{10}\right)$ in our sample could likely follow this latter evolution path. Indeed, we see that the galaxies in our sample that show extended structure at low redshifts $(z<0.3)$ are among the most massive of the sample.

The conclusions of Koo et al. (1995) and Guzmán et al. (1998) rest partially on the assumption that galactic winds from the last starburst event will remove the remaining gas from these systems and halt star formation, causing them to fade several magnitudes by $z=0$. Through modeling the UV and optical broadband colors we find that about half the galaxies in our present sample could have formed at most $\sim 10 \%$ of their stellar mass in bursts within the past $100 \mathrm{Myr}$, and only 15 (all having $M_{\star}<10^{9}$ ) could have formed over $20 \%$ of their stellar mass within the last $100 \mathrm{Myr}$. If the last starburst event removes all remaining gas from these systems, as Koo et al. (1995) proposed, then why did the previous star-forming events in these galaxies that produced the majority of the stars in these systems not halt star formation? Our derived star formation histories of these galaxies argues that the current star formation events will not halt star formation in every galaxy. But what percentage of these will continue to experience further bursts of star formation is unknown. However, even in the lowmass systems of the present sample, residual star formation or another burst of star formation is ultimately unlikely to change the evolutionary outcome proposed by Koo et al. (1995) and Guzmán et al. (1998) for the most compact low-mass galaxies in our sample. We suggest that the more extended sources of our sample are more likely to become present-day dwarf irregulars rather than dwarf spheroidals.

\section{SUMMARY}

We have presented DEIMOS spectra for objects detected by GALEX in the Medium Imaging Survey (MIS) survey with imaged counterparts in SDSS; a total exposure time of 30 minutes per slit mask was used. GALEX has proven to be a sensitive instrument for wide-field galaxy surveys. We have shown that the GALEXMIS followed up with a 30 minute integration with Keck DEIMOS yields redshifts and line measurements for star-forming galaxies to $z \sim 0.7$, and has yielded four QSOs, three of which are previously uncataloged. The matched sample reaches approximately 3 mag fainter in $r$ than the SDSS spectroscopic survey limits. The sample is not a homogenous sample but is indicative of the types of galaxies forming stars out to $z \sim 0.7$. We have derived physical parameters for these galaxies from the SEDs and compared this sample to a sample of 50,000 SDSS galaxies with GALEX detections.

1. We find that roughly one-third of the galaxies are starforming late-type disk galaxies, four are QSOs at $z>1$, and the remaining galaxies are faint blue low-mass starbursts.

2. Approximately 3 out of 14 star-forming galaxies show emission-line ratios indicative of an AGN. A similar fraction was found by Sullivan et al. (2000) in their UV-selected sample.

3. The masses of the galaxies are typically lower than what is found locally in the SDSS spectroscopic sample. The range of $M_{\star}$ for the DEIMOS sample spans from $10^{8}$ to $10^{11} M_{\odot}$, whereas the median of the SDSS spectroscopic sample is $\sim 5 \times 10^{10}$.

4. The SFRs of the galaxies at $z>0.25$ are roughly an order of magnitude greater than the SFRs for the galaxies at $z<0.25$.

5. Besides three of the most massive and reddest in NUV $-r$ color, the remaining galaxies show evidence of a starburst in the last $100 \mathrm{Myr}$, with $\log b \sim-0.1$. Fifteen of the galaxies in the lower mass range of the sample could have formed more than $20 \%$ of their stellar mass in bursts of star formation within the last $100 \mathrm{Myr}$.

6. Our sample has similar velocity dispersions, SFRs, and $B$ luminosities to previous samples of faint blue galaxies, although the galaxies in our present study are 2 mag fainter in surface brightness.

GALEX is a NASA Small Explorer, launched in 2003 April. We gratefully acknowledge NASA's support for construction, operation, and science analysis for the GALEX mission, developed in cooperation with the Centre National d'Etudes Spatiales of France and the Korean Ministry of Science and Technology. The authors wish to recognize and acknowledge the very significant cultural role and reverence that the summit of Mauna Kea has always had within the indigenous Hawaiian community. We are most fortunate to have the opportunity to conduct observations from this mountain. The analysis pipeline used to reduce the DEIMOS data was developed at University of California, Berkeley, with support from NSF grant AST 00-71048. The authors thank an anonymous referee for extremely helpful comments.

Facilities: GALEX, Keck:II 
Abazajian, K., et al. 2004, AJ, 128, 502

Baldwin, J. A., Phillips, M. M., \& Terlevich, R. 1981, PASP, 93, 5

Bertin, E., \& Arnouts, S. 1996, A\&AS, 117, 393

Blanton, M., et al. 2003, AJ, 125, 2276

Brinchmann, J., Charlot, S., White, S. D. M., Tremonti, C., Kauffmann, G. Heckman, T., \& Brinkmann, J. 2004, MNRAS, 351, 1151

Bruzual, G., \& Charlot, S. 2003, MNRAS, 344, 1000

Charlot, S., \& Fall, S. M. 2000, ApJ, 539, 718

Ellis, R. S. 1997, ARA\&A, 35, 389

Faber, S. M., et al. 2003, Proc. SPIE, 4841, 1657

Fukugita, M., Ichikawa, T., Gunn, J. E., Doi, M., Shimasaku, K., \& Schneider, D. P. 1996, AJ, 111,1748

Guzmán, R., Jangren, A., Koo, D. C., Bershady, M. A., \& Simard, L. 1998, ApJ, 495, L13

Guzmán, R., Koo, D. C., Faber, S. M., Illingworth, G. D., Takamiya, M., Kron, R. G., \& Bershady, M. A. 1996, ApJ, 460, L5

Guzmán, R., et al. 1997, ApJ, 489, 559

Hammer, F., Gruel, N., Thuan, T. X., Flores, H., \& Infante, L. 2001, ApJ, 550, 570

Hogg, D. W., Finkbeiner, D. P., Schlegel, D. J., \& Gunn, J. E. 2001, AJ, 122, 2129

Kauffmann, F., et al. 2003, MNRAS, 341, 33

Kennicutt, R. C., Jr. 1998, ARA\&A, 36, 189

Kewley, L. J., Dopita, M. A., Sutherland, R. S., Heisler, C. A., \& Trevena, J. 2001, ApJ, 556, 121

Kobulnicky, H. A., \& Kewley, L. 2004, ApJ, 617, 240

\section{REFERENCES}

Koo, D. C., Guzmán, R., Faber, S. M., Illingworth, G. D., Bershady, M. A., Kron, R. G., \& Takamiya, M. 1995, ApJ, 440, L49

Mallen-Ornelas, G., Lilly, S. J., Crampton, D., \& Schade, D. 1999, ApJ, 518, L83

Martin, C. D., et al. 2005a, ApJ, 619, L1 2005b, ApJ, 619, L59

Milliard, B., Donas, J., Laget, M., Armand, C., \& Vuillemin, A. 1992, A\&A, 257, 24

Morrissey, P., et al. 2005, ApJ, 619, L7

Osterbrock, D. E. 1989, Astrophysics of Gaseous Nebulae and Active Galactic Nuclei (Mill Valley: University Science)

Phillips, A. C., et al. 1997, ApJ, 489, 543

Pier, J. R., Munn, J. A., Hindsley, R. B., Hennessy, G. S., Kent, S. M., Lupton, R. H., \& Ivezic, Z. 2003, AJ, 125, 1559

Salim, S., et al. 2005, ApJ, 619, L39 2007, ApJS, 173, 267

Scalo, J. M. 1986. Fundam. Cosmic Phys., 11, 1

Schiminovich, D., et al. 2005, ApJ, 619, L47

Schlegel, D. J., Finkbeiner, D. P., \& Davis, M. 1998, ApJ, 500, 525

Smith, J. A., et al. 2002, AJ, 123, 2121

Stoughton, C., et al. 2002, AJ, 123, 485

Strauss, M. A., et al. 2002, AJ, 124, 1810

Sullivan, M., Treyer, M. A., Ellis, S. R., Bridges, T. J., Milliard, B., \& Donas, J. 2000, MNRAS, 312, 442

Treyer, M. A., et al. 2005, ApJ, 619, L19

Wyder, T. K., et al. 2005, ApJ, 619, L15 